\title{
Analysis of Model Parameters for a Polymer Filtration Simulator
}

\author{
N. Brackett-Rozinsky, ${ }^{1}$ S. Mondal, ${ }^{2}$ K. R. Fowler, ${ }^{2}$ and E. W. Jenkins ${ }^{3}$ \\ ${ }^{1}$ UCLA Department of Mathematics, University of California Los Angeles, Los Angeles, CA 90095-1555, USA \\ ${ }^{2}$ Department of Mathematics, Clarkson University, Potsdam, NY 13699-5815, USA \\ ${ }^{3}$ Department of Mathematical Sciences, Clemson University, Clemson, SC 29634, USA \\ Correspondence should be addressed to K. R. Fowler, kfowler@clarkson.edu
}

Received 30 March 2011; Revised 28 July 2011; Accepted 14 August 2011

Academic Editor: MuDer Jeng

Copyright (๑) 2011 N. Brackett-Rozinsky et al. This is an open access article distributed under the Creative Commons Attribution License, which permits unrestricted use, distribution, and reproduction in any medium, provided the original work is properly cited.

\begin{abstract}
We examine a simulation model for polymer extrusion filters and determine its sensitivity to filter parameters. The simulator is a three-dimensional, time-dependent discretization of a coupled system of nonlinear partial differential equations used to model fluid flow and debris transport, along with statistical relationships that define debris distributions and retention probabilities. The flow of polymer fluid, and suspended debris particles, is tracked to determine how well a filter performs and how long it operates before clogging. A filter may have multiple layers, characterized by thickness, porosity, and average pore diameter. In this work, the thickness of each layer is fixed, while the porosities and pore diameters vary for a two-layer and three-layer study. The effects of porosity and average pore diameter on the measures of filter quality are calculated. For the three layer model, these effects are tested for statistical significance using analysis of variance. Furthermore, the effects of each pair of interacting parameters are considered. This allows the detection of complexity, where in changing two aspects of a filter together may generate results substantially different from what occurs when those same aspects change separately. The principal findings indicate that the first layer of a filter is the most important.
\end{abstract}

\section{Introduction}

Filters are a key component in many science and engineering disciplines and industrial applications. They exist to filter debris from a variety of fluids, including polymers, blood, oil, and air, and to separate components of distinct fluids from each other, as water from gasoline. In this paper, we study the behavior of an extrusion filter located within a fiber-spinning process (see Figure 1). Solid polymer pellets are melted into a fluid, and the fluid is pushed through an extrusion die before being spun into a fiber. The finished fiber product must be consistent in quality; hence, the polymer melt used to produce the fiber must be sufficiently pure. This means that any debris, whether from unmelted pellets or foreign objects introduced during the flow, must be trapped by the filter. Consistent fiber quality also requires that the polymer fluid be pushed through the spinneret, and hence the filter, at a constant rate. As debris deposits within the filter, the metering pump must run at increased pressure to ensure this constant flow rate. The result is an increase in the pressure drop across the filter, which is measured during the fiberspinning process. Once the pressure drop reaches a specified threshold value, the filter is replaced. Continuing to allow the process to operate above the threshold value will eventually damage the metering pump.

As part of a larger effort at the Center for Advanced Engineering Fiber and Films (CAEFF) [1] to simulate a complete fiber-spinning process [2], researchers developed a three-dimensional filter simulation tool [3]. The numerical model was developed to help process engineers understand the mechanisms behind debris removal, and previous work indicates that the simulated values for pressure drop are in agreement (to within 10\%) with empirical data [3]. In this work, we make use of the simulation tool to study, in an optimization framework, the importance of certain design parameters on selected performance measures. We base our performance measures on two ideas: effective removal of debris particles and prolonged filter lifetime. Filter replacement is costly, often requiring that the entire spinline be taken out of production. The introduction of in-line filters 


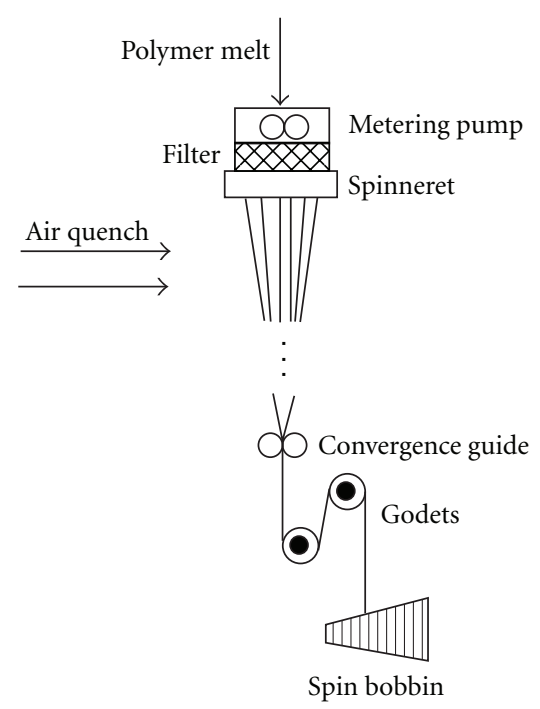

FIgURE 1: Fiber melt-spinning process.

is not believed to completely eliminate this problem, as some analysts see these filters in combination with conventional filters still located at the spinneret [4]. At a minimum, replacement of all conventional filters with in-line filter systems will take time based on the costs involved with replacement [5].

The performance measures we use lead to competing objectives, as extending the filter lifetime could be achieved by simply reducing the amount of trapped debris. In our previous work, we have evaluated optimal filter performance using a multiobjective genetic algorithm $[6,7]$. We have also combined the competing objective into a single-objective functional using barrier and penalty methods [8,9], studying both one- and two-layer filter designs. The one-layer results obtained in [8] compared favorably with those in [6] and the two-layer results seemed reasonable from an intuitive and engineering perspective, as the optimal configuration matched the design principles given in the engineering literature [10].

For each of those above studies, the objective function is defined in terms of output from the simulator. The model for the filtration process includes a system of coupled partial differential equations for the polymer flow through the filter along with empirical tools that describe the amount of debris in the polymer and the probability that a debris particle is retained by the filter. In this regard, the important considerations are how long the filter lasts, how much debris escapes from it, and how quickly the pressure drop rises.

However, there were competitive combinations of certain initial configurations and objective functionals that lead to designs that were suboptimal, from an engineering standpoint [8]. In other cases, even with optimal configurations, the parameters were pushed to the edge of the design window. To better understand these findings, and to assist in drafting an optimal filter design, we need to determine which parameters contribute significantly to our performance measures. Towards this end, we obtained data using the filter simulation tool and sampled a variety of filter configurations. These data provide guidance towards optimal design parameters and insight into the effects of such parameters on the behavior of the filter.

We begin by considering a filter with two layers and perform a qualitative analysis on the simulation output. We look at slices of its design space made by fixing two parameters and varying two parameters. This provides unexpected results suggesting that the first layer is the only important one for designing the filter. Although that could explain the previously mentioned filter that an optimizer came up with, having larger pores in the second layer than the first, it was an unsatisfactory outcome. To uncover more details, we advance to the analysis of three-layer filters.

For the three-layer configuration, we apply the statistical technique called analysis of variance (ANOVA) to identify significant factors. Specifically, we use the Kruskal-Wallis test. This test, as with all ANOVA tests, looks at the variance of some response function, in this case the filter performance, within several groups of data points, and the variance between those groups of data. By comparing those two variances, the test determines whether there are significant differences between groups, or whether the groups are statistically indistinguishable with regard to the response function values. An advantage of this approach is that it provides a framework for a variety of simulation methods, including the three-dimensional algorithm we apply here.

We proceed by describing the simulation tool in Section 2 and the measures of filter performance that are based on simulation output in Section 3. We provide analyses of the two- and three-layer filters, focusing on how the pore diameter and porosity in each layer impact the filter performance metrics in Sections 4 and 5, and we summarize these findings and point the way towards future work in Section 6.

\section{Debris Deposition Model}

The model for the filtration code (i.e., the "simulator") was originally developed in [11] in a one-dimensional setting. The work was extended to three spatial dimensions in [3], which lead directly to the computational tool we use in this paper. The material entering the filter consists of molten polymer, unmelted polymer gel particles (formed when the polymer does not completely melt in the heating/mixing stage), and other debris such as metal particles. We assume that the density of the debris is negligible in comparison to the polymer density, and that the filter thickness is small enough to allow gravitational forces to be ignored $[3,11]$.

The flow through the filter is governed by the continuity equation (mass conservation) and Darcy's Law, modified to account for the non-Newtonian behavior of the polymer melt. Darcy's Law, empirically derived in 1856, defines a Darcy velocity associated with porous media flow. This averaged, or upscaled, velocity $\mathbf{u}$ is proportional to the pressure gradient, so that

$$
\mathbf{u}=\frac{k}{\mu_{\mathrm{eff}}} \nabla p,
$$


where $\mu_{\text {eff }}$ is the effective fluid viscosity $[12,13]$, and $k$ is the relative permeability of the porous medium. The effective viscosity depends on the power law index of the fluid, which is a measure of the shear-thinning behavior of the fluid, and the magnitude of the velocity of the fluid. (The power law index is 1 for Newtonian, or non-shear-thinning, fluids.)

The permeability of a porous medium is related to the ability of the medium to transmit fluids. The filter becomes less efficient at fluid transport as debris is deposited; thus, any function that models permeability must decrease as porosity decreases. The permeability parameter $k$ is currently modeled using a Blake-Kozeny relationship that depends on the average pore size, $d_{p}$, and the current filter porosity, $\eta$. This relationship for $k$, derived in [14] and used in $[3,11]$, is

$$
k=\frac{d_{p}^{2} \eta^{3}}{150(1-\eta)^{2}} .
$$

The permeability is calculated at the end of every time step, using the updated values for $d_{p}$ and $\eta$. The mass flow rate is kept constant during the simulation; therefore, as $k$ decreases, the pressure drop across the filter must increase. The large values of pressure required at the inlet may lead to damage of the metering pump, so the filter must be replaced once the pressure drop reaches a threshold value. The threshold value is set at $35 e 6 \mathrm{~Pa}$ in the simulator, which is comparable to existing industry thresholds [3]. The flow equation is discretized using cell-centered finite differences, which is known to conserve mass locally [15]. The resulting system of nonlinear equations is solved using a fixed-point iteration scheme.

The debris entering the filter is specified in terms of a mass fraction relative to the mass of the mixture. The distribution of debris particles is characterized statistically, as in [4], using either truncated or standard normal probability density functions [6]. We use three material types in the simulator, each characterized by a different probability distribution based on empirical data collected on the materials in [16]. The means and standard deviations of the distributions are input variables, and a user may choose either a lefttruncated, right-truncated, or full normal distribution as their debris profile.

Debris is tracked as it travels through each computational cell. Fluxes are calculated on each cell face and used to determine the amount of available debris that is transported into a given cell. Debris particles with a larger diameter than the pore diameter associated with the cell are eligible for capture. The percentage of these particles retained by the filter is determined by an empirical retention function associated with the filter [3]. The retention function is dependent on the initial pore diameter, so the characteristics of the retention function change as the optimizer selects new points to evaluate. All particles not captured within a computational cell are transported to downstream cells. The porosity of the filter and the average pore diameter of the filter are updated at the end of every time step using the information on the mass of the deposited debris.

Filters can be made from sand, sintered metal, compressed with sufficient force to produce a cake material, or layers of wired mesh, with mesh spacings small enough to trap particles a few microns in diameter. A filter pack often contains multiple layers, each of which is characterized by a porosity, an average pore diameter, and a retention function. Filters are usually designed with smaller pore diameters in lower layers [10]. The simulator can handle up to five distinct filter layers, but we only consider two- and three-layer filters in this work. We can obtain filter lifetimes, amount of trapped debris, amount of escaped debris, and pressure drop values as output from the simulator. More detailed information on the model can be found in $[3,6,17]$.

\section{Measures of Filter Performance}

This study focuses on the porosity $\eta_{i}$ and average pore diameter $d_{p i}$ for layers $i=1,2$, or 3 and how they impact filter performance. The objective functions described below measure filter lifetimes in different manners. The first measures the lifetime using the number of hours before the pressure drop threshold is reached. The second objective function measures lifetime using slope of the pressure curve (see Figure 2). Previous optimization studies attempted to increase lifetime by decreasing the sharp increase in the behavior of the curve towards the end of the filter lifetime [7-9]. Let $p_{n}$ denote the difference in pressure (in pascals) across the filter at the $n$th time step $t_{n}$. One approach to measure the "flatness" of the pressure drop curve is to use a linear estimate of the total change in pressure over the lifetime of the filter. Suppose the lifetime of the filter consists of $N$ time steps. The total change in pressure can be modeled by the line passing through the points $\left(t_{0}, p_{0}\right)$ and $\left(t_{N}, p_{N}\right)$. A reasonable measure is the slope of this line, which can be expressed as

$$
m=\frac{p_{N}-p_{0}}{t_{N}-t_{0}}
$$

where $m$ has units of pascals per hour. The pore spaces in the filter are continuously being occupied by debris; thus, both in theory and in the behavior of the simulator, $p_{i}>p_{i-1}$, making $m$ strictly positive. We refer to $m$ in the following discussions as the secant objective, since it is the slope of the secant line between the pressures at initial and final times of the simulation. We also consider the amount of debris that escapes the filter as the third measure of filter performance.

\section{Two-Layer Analysis}

Industrial polymer extrusion filters commonly use a threelayer design. This is based on the concept of trapping large debris first, then smaller and smaller particles further along. The principle behind this approach is that collecting all the foreign material in one place would quickly clog the filter, whereas spreading it out improves fluid flow and thus the lifetime of the filter. To better understand the behavior of a multilayer filter design, we begin by considering a filter with two layers. Intuitively, one expects the first layer to have a coarser mesh and the second layer to have a finer mesh. The issue of designing two-layer filters was addressed in 


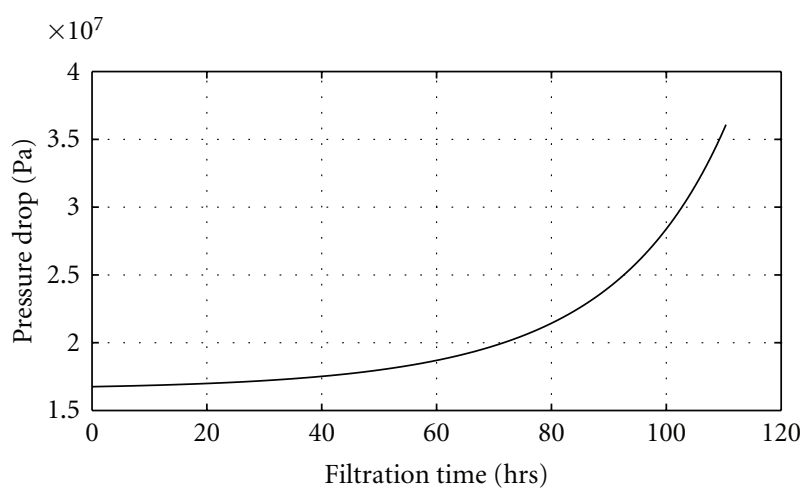

FIGURE 2: Representative pressure drop curve.

previous papers [7-9]. There are a variety of derivative-free optimization methods used to find competitive parameter values.

In [9], the quality of a filter was measured by a unified objective function that rewards higher lifespans and penalizes for the amount of debris that does not get trapped. In that simulator-driven optimization procedure, a number of distinct filter designs with comparable objective scores were identified. Contrary to a priori expectations, some of these competitive designs had finer meshes in the first layer and coarser meshes in the second layer. These "upsidedown" results prompted inquiry as to which parameters have significant effects on which aspects of the objective function. The discovery of multiple disparate filter designs with similarly high quality indicated that there may be substantial interaction between parameters. This type of interaction, where the effect of changing one variable depends strongly on the values of other variables, is common among complex systems and demonstrates nonlinearity.

In order to identify sources of significant effects on the objective functions, filter designs across the parameter space are evaluated by the simulator. For two-layer filters, by fixing two components in the vector $x$ of parameters and allowing the others to vary, a two-dimensional slice of the parameter space is obtained. Selecting values of the nonfixed parameters regular intervals forms a grid filter designs that can be viewed as "landscape" surfaces in three dimensions. In this treatment, four slices were made for the lifetime, secant, and amount of escaped debris.

In the first slice, the porosities of both layers are fixed with $\eta_{1}=\eta_{2}=0.62$, meaning that a cross-sectional slice of the filter will have, on average, $62 \%$ of its area open for fluid to pass through. The average pore diameters of both layers vary from 23 to $55 \mu \mathrm{m}$. The resulting landscape plots are shown in Figure 3. It can be seen that the values of all three objective functions vary greatly as the average pore diameter in layer one changes, and scarcely at all as the average pore diameter in layer two changes. This would suggest that in the simulator, the amount of debris that gets out of layer one into layer two is not only insufficient to clog layer two, but also has a negligible effect on preventing layer one from clogging by its absence.
In the second slice (Figure 4), the average pore diameter of the first layer is fixed at $35 \mu \mathrm{m}$, and the average pore diameter of the second layer is fixed at $24 \mu \mathrm{m}$. The average porosities of both layers vary from 0.1 to 0.7 . The resulting landscape plots are shown below. These landscapes show a clear dependence of all three objective functions on the porosity of layer one. They also show that the porosity of layer two has almost no effect.

In the third slice (Figure 5), the porosity of the second layer is fixed at 0.65 , and the average pore diameter of the second layer is fixed at $24 \mu \mathrm{m}$. The average porosity of layer one varies from 0.1 to 0.7 and the average pore diameter of layer one varies from 23 to $55 \mu \mathrm{m}$. The resulting landscape plots are shown below. These landscapes show a clear dependence of all three objective functions on both the porosity and pore diameter of layer one, as expected from the previous two slices.

In the fourth slice (Figure 6), the porosity of the first layer is fixed at 0.65 , and the average pore diameter of the first layer is fixed at $38 \mu \mathrm{m}$. The average porosity of layer two varies from 0.1 to 0.7 and the average pore diameter of layer two varies from 23 to $55 \mu \mathrm{m}$. The resulting landscape plots are shown below.

From all four sets of landscape figures, it becomes apparent upon inspection that the second layer seems insignificant. The lifetime and rate of pressure change depend on porosity of the first layer, and the amount of debris that escapes depends on the average pore diameter of the first layer. One might suppose that escaped debris should depend on the smallest average pore diameter for any layer, but that does not appear to be the case in this simulation model. To determine precisely what happens and why these results arise, we investigate a full three-layer filter design with a rigorous statistical analyses.

\section{Three-Layer Analysis}

We now consider $\eta_{1}, d_{p 1}, \eta_{2}, d_{p 2}, \eta_{3}$, and $d_{p 3}$ as parameters for a sensitivity analysis of the three-layer filter. This is done by sampling filter parameters across the design space and evaluating each of them with the simulator to generate the data for the ANOVA. Using the sampled data, we form quintiles of each design parameter. By arranging the data in quintile groups, the ANOVA determines whether any changes in that parameter bring about significant effects in the objective functions. Main effects of each parameter are determined, as are interaction effects of two parameters jointly, for the three metrics of filter quality. By performing these statistical tests and analyses, we shed light on how best to design a polymer extrusion filter. In particular, multiple comparison tests reveal which quintiles of each parameter have significant effects on filter performance. Those results, combined with interaction plots, provide new detail that can be incorporated into future optimization work.

5.1. Grouping Parameters. In order to obtain results from all regions of the parameter space, a total of 360 filter designs were chosen through a Latin-hypercube method. Using a Latin hypercube ensures a uniform distribution of selections. 


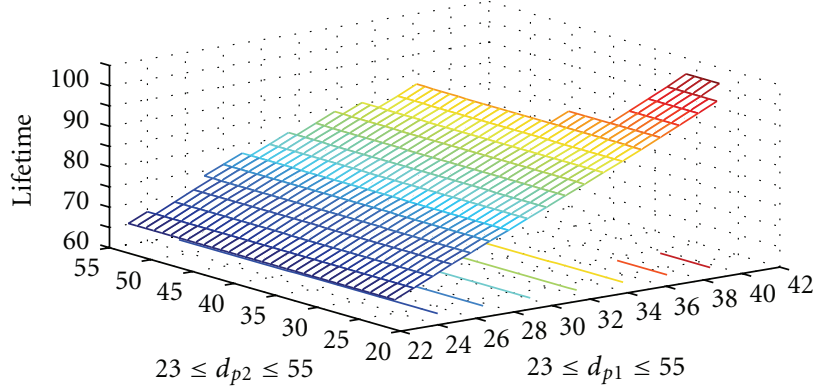

(a) Lifetime

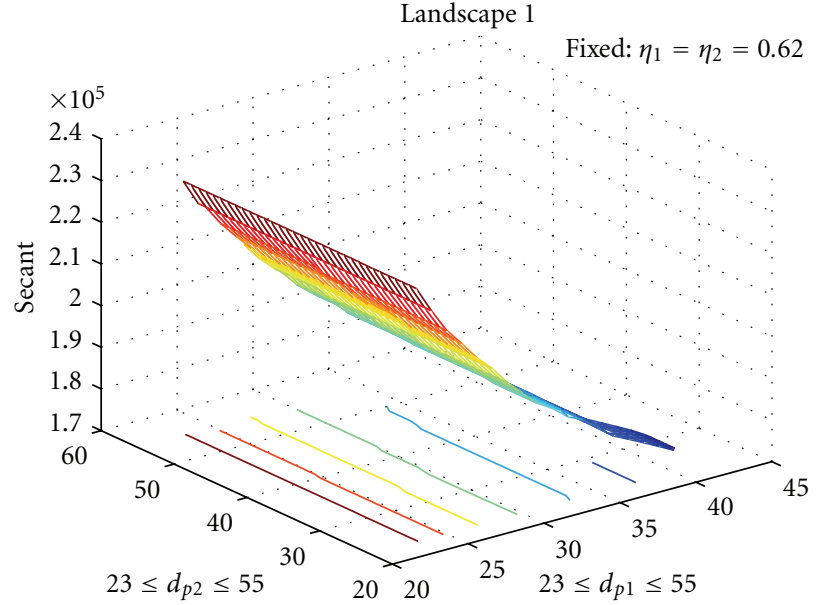

(b) Secant

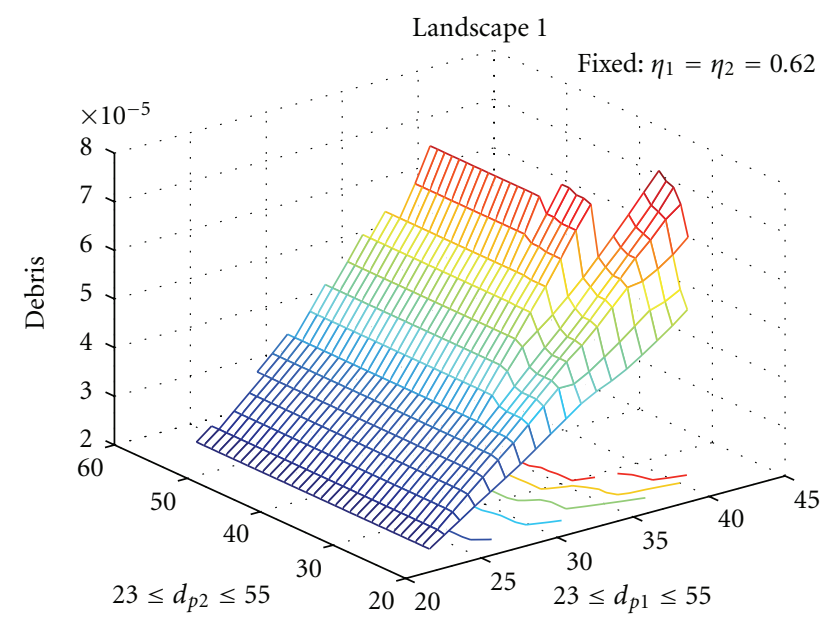

(c) Debris

FIGURE 3: Landscapes with $\eta_{1}=\eta_{2}=0.62$ while $d_{p 1}, d_{p 2}$ vary.

Each parameter's range is divided into intervals, thus partitioning the whole design space into a six-dimensional grid, where each subsection corresponds to one interval of every variable. Subsections are chosen, so no two chosen sections are on the same axis-aligned hyperplane. This guarantees a broad spectrum of parameter combinations while keeping the requisite number of simulations manageable. Specific values are picked at random from within the selected grid cells, ensuring that the designs have some natural irregularity. The 360 designs were chosen in this manner, and simulation results were obtained for each design. The solution algorithm in the simulator program failed on 41 of these designs. The remaining 319 "successful" simulations provide the data for subsequent analyses.

With respect to each parameter, the lowest 64 values went into the first quintile, the next-lowest 64 values into the second quintile, the middle 64 values into the third quintile, the next highest 64 values into the fourth quintile, and the highest 63 values into the fifth quintile. Note that the quintile groupings are independent. That is to say, the specific filter designs for which the values of one parameter were among the lowest quintile are by no means, and in fact were specifically chosen not to be, the same designs for which another parameter has all its values in one quintile. The values of all parameters besides the one that is being considered are spread across their ranges. Table 1 shows those ranges of values for each parameter in each quintile, which we refer to according to the group numbers.

5.2. One-Way ANOVA. The one-way ANOVA method compares the variance of the objective function within each group to that same variance between groups. If this ratio is sufficiently small for a given parameter, it constitutes evidence that the objective function is sensitive to changes in that parameter. The ANOVA test generates a $P$ value, which is used to establish a confidence level for that sensitivity. As employed here, a $P$ value less than 0.05 implies $95 \%$ confidence that the response is sensitive to the corresponding parameter. For this study, because of the nearly uniform sample selection method, and due to the fact that the individual data groups come from the same larger population, the distribution of values of each parameter is approximately 


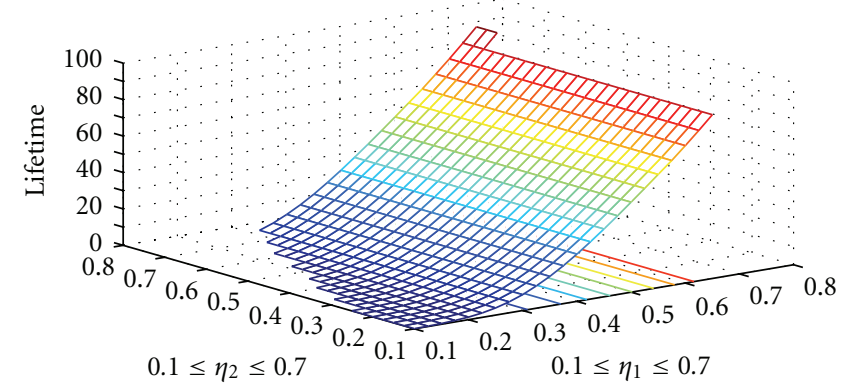

(a) Lifetime

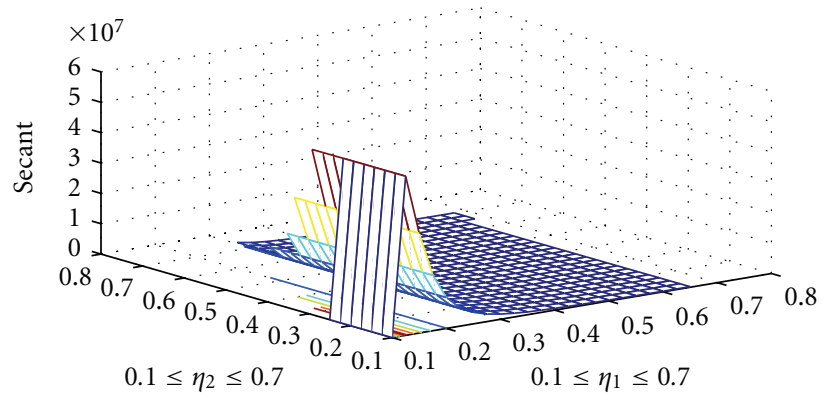

(b) Secant

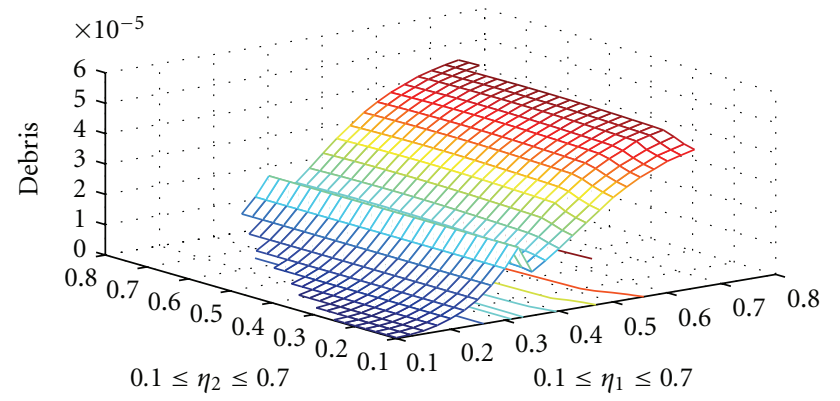

(c) Debris

FIGURE 4: Landscapes with $d_{p 1}=35 \mu \mathrm{m}, d_{p 2}=24 \mu \mathrm{m}$ while $\eta_{1}, \eta_{2}$ vary.

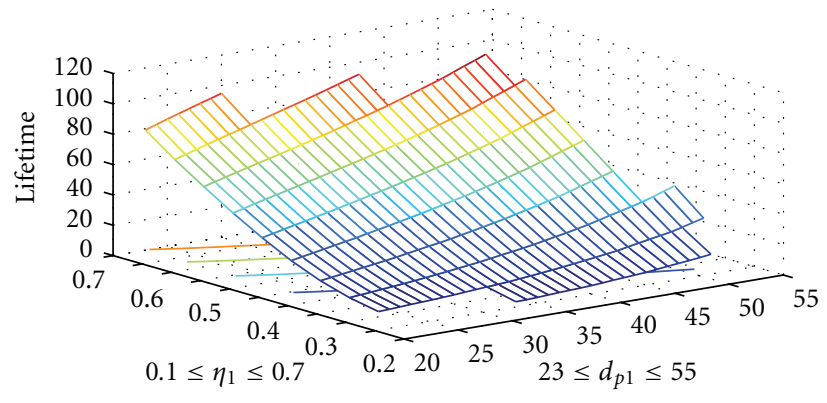

(a) Lifetime

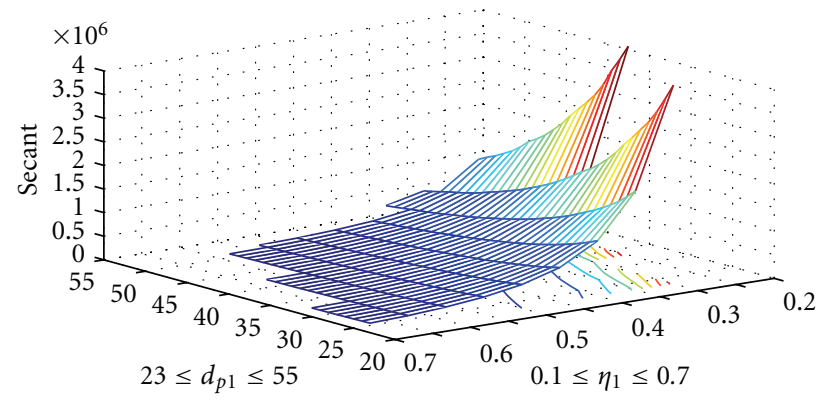

(b) Secant

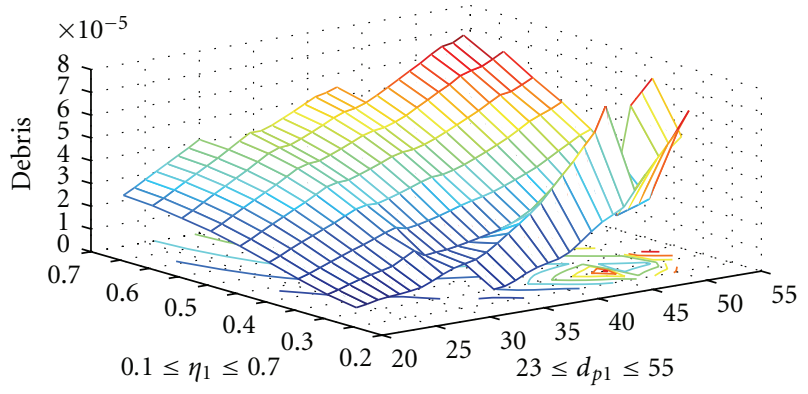

(c) Debris

FIGURE 5: Landscapes with $\eta_{2}=0.65, d_{p 2}=24 \mu \mathrm{m}$ while $\eta_{1}, d_{p 1}$ vary.

uniform. In particular, the distribution within each quintile group is demonstrably not normal, as verified by the Lilliefors normality test implemented by MATLAB. Therefore, it is necessary to use nonparametric ANOVA, in this case the Kruskal-Wallis test, to calculate sensitivity and main effects. Table 2 shows the $P$ values for main effects, from the
Kruskal-Wallis test. This reveals that the relevant factors are the porosity and pore diameter of layer one for all objectives, and the pore diameter of layer two for the debris objective.

These results show that the first layer has far more significant effects on the simulated filter performance relative to these performance measures than do the other layers. 


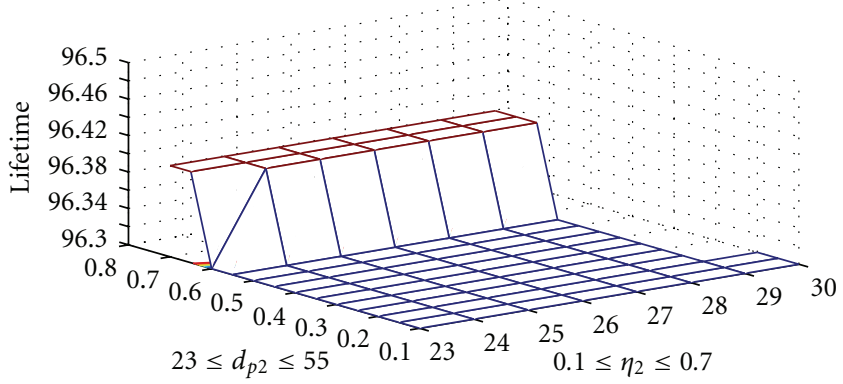

(a) Lifetime

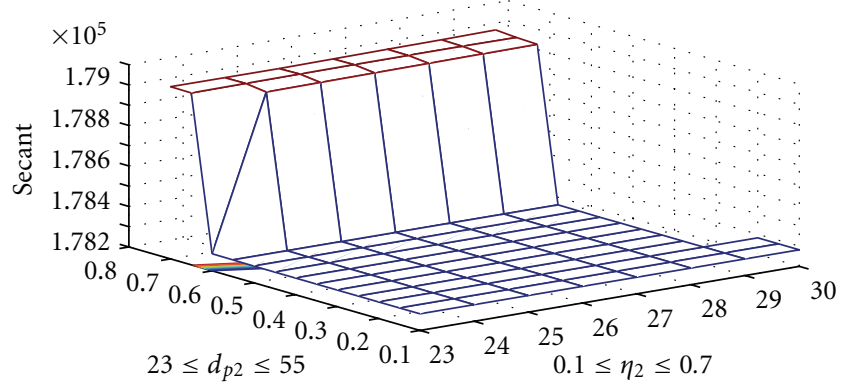

(b) Secant

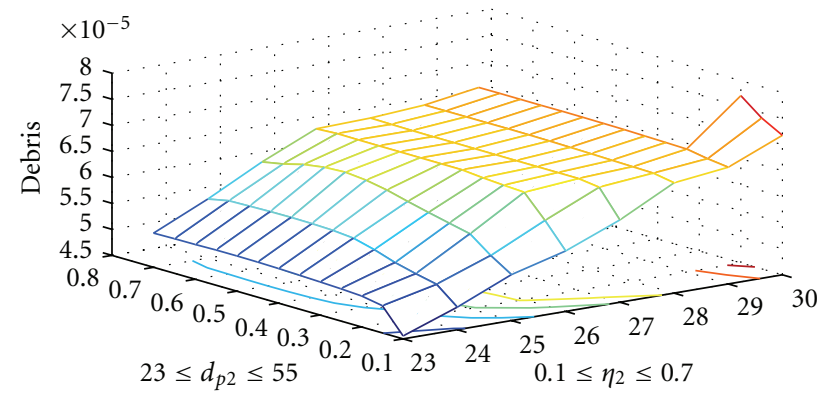

(c) Debris

FIGURE 6: Landscapes with $\eta_{1}=0.65, d_{p 1}=38 \mu \mathrm{m}$ while $\eta_{2}, d_{p 2}$ vary.

TABLE 1: The range of each parameter in each of its groups.

\begin{tabular}{lcccccc}
\hline Group & $\eta_{1}$ & $d_{p 1} \mu \mathrm{m}$ & $\eta_{2}$ & $d_{p 2} \mu \mathrm{m}$ & $\eta_{3}$ \\
\hline 1 & $0.4008-0.4576$ & $23.083-29.205$ & $0.4003-0.4536$ & $23.016-28.751$ & $0.4006-0.4595$ & $23.019-29.481$ \\
2 & $0.4587-0.5130$ & $29.251-35.266$ & $0.4542-0.5082$ & $28.789-34.604$ & $0.4606-0.5207$ & $29.491-35.919$ \\
3 & $0.5140-0.5676$ & $35.334-41.731$ & $0.5088-0.5624$ & $34.665-40.666$ & $0.5211-0.5821$ & $35.993-42.524$ \\
4 & $0.5688-0.6309$ & $41.812-48.312$ & $0.5630-0.6166$ & $40.751-46.986$ & $0.5826-0.6392$ & $42.599-48.682$ \\
5 & $0.6322-0.6994$ & $48.408-54.933$ & $0.6172-0.6976$ & $47.135-54.973$ & $0.6395-0.7000$ & $48.759-54.930$ \\
\hline
\end{tabular}

TABle 2: The $P$ values for sensitivity of each objective.

\begin{tabular}{lccc}
\hline Kruskal-Wallis $P$ value & Lifetime & Secant & Debris \\
\hline$\eta_{1}$ & $2.02 \mathrm{E}-37$ & $6.55 \mathrm{e}-34$ & $1.04 \mathrm{e}-3$ \\
$d_{p 1}$ & $6.34 \mathrm{E}-24$ & $2.73 \mathrm{e}-27$ & $1.75 \mathrm{e}-55$ \\
$\eta_{2}$ & 0.614 & 0.524 & 0.372 \\
$d_{p 2}$ & 0.769 & 0.742 & $2.71 \mathrm{e}-4$ \\
$\eta_{3}$ & 0.589 & 0.722 & 0.998 \\
$d_{p 3}$ & 0.676 & 0.708 & 0.164 \\
\hline
\end{tabular}

This can be visualized by main effects plots, which show the average response function values per group. Figure 7 shows two representative main effects plots for the lifetime objective. The plot on the left reflects strong dependence of lifetime on the porosity in the first layer, while on the right, the filter lifetime with respect to the porosity in the second layer is relatively flat.

5.3. Multiple Comparison ANOVA. We have identified the sensitive parameters using the one-way ANOVA method. Then, we determine which group of each sensitve parameter is different from the other. We use the multiple comparison tests after controlling the experiment-wise error rate. The experiment-wise error rate in making pairwise comparisons among $k$ groups is the probability of declaring at least two groups to be different when there are no differences among the $k$ groups. To keep the experiment-wise error rate no greater than $\alpha$ (our level of significance is 0.05 ), the Bonferroni adjustment is to do each of the $\left(\begin{array}{l}k \\ 2\end{array}\right)$ comparisons at level of significance

$$
\alpha^{\prime}=\frac{\alpha}{\left(\begin{array}{l}
k \\
2
\end{array}\right)} \text {. }
$$

In Table 3, we show the results for the pore diameter in layer one $\left(d_{p 1}\right)$ with respect to the filter lifetime objective. Values of 1 correspond to $95 \%$ confidence that two groups are significantly different in effect from one another, whereas values of 0 indicate they are not. Thus, we can conclude that all the groups are significantly different from each other except the pairs of groups $(1,2),(2,3)$, and $(4,5)$. The results for the porosity in layer one $\left(\eta_{1}\right)$ with respect to the lifetime showed different results in that all groups were significantly 


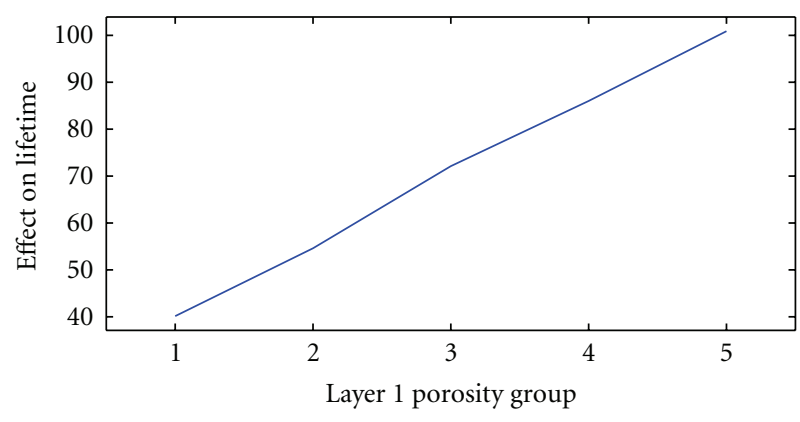

(a) Main effects for porosity in layer 1

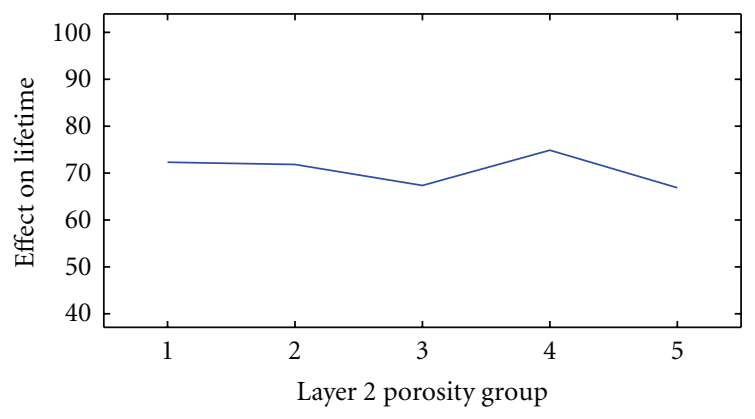

(b) Main effects for porosity in layer 2

FIGURE 7: Main effects plots for the lifetime objective for $\eta_{1}$ and $\eta_{2}$.

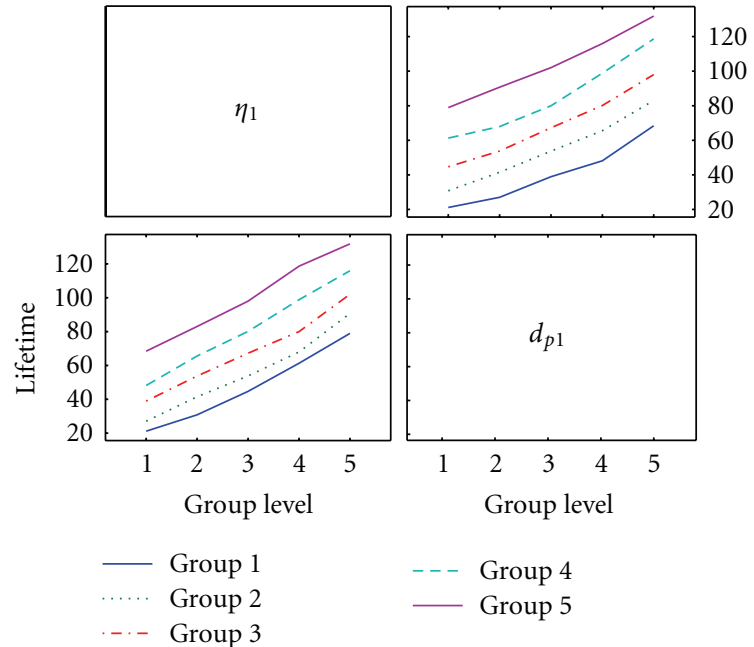

(a) Interaction plots for lifetime and $\eta_{1}, d_{p 1}$

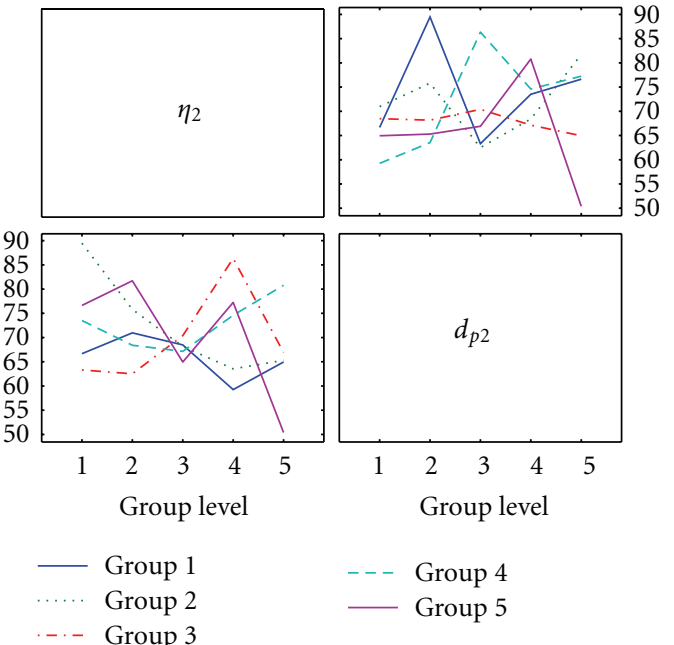

(b) Interaction plots for lifetime and $\eta_{2}, d_{p 2}$

FIgURE 8: Interaction plots for filter lifetime for parameters in layers 1 and 2. The legends are the same for both plots.

TABLe 3: Pairs of groups for $d_{p 1}$ for which the filter lifespans differ significantly from each other.

\begin{tabular}{lcccc}
\hline$d_{p 1}$ & Group 2 & Group 3 & Group 4 & Group 5 \\
\hline Group 1 & 0 & 1 & 1 & 1 \\
Group 2 & & 0 & 1 & 1 \\
Group 3 & & & 1 & 1 \\
Group 4 & & & & 0
\end{tabular}

different from each other except between groups 3 and 4 . For the secant objective, the porosity and pore diameter also displayed significant differences across all pairs of groups except for between group pairs $(3,4)$ and $(4,5)$ for $\eta_{1}$ and between group pairs $(1,2)$ and $(2,3)$ for $d_{p 1}$.

For the debris objective, there was a significant difference between all pairs of groups with respect the pore diameter in layer one. This was not true for the porosity in layer one, which only showed a significant group 1 with groups 3,4 and 5. It is noteworthy that the only significant parameters for any layers beyond the first are with regard to escaped debris. For the pore diameter in layer two- and the debris
TABLE 4: Pairs of groups for $d_{p 2}$ for which the escaped debris differ significantly from each other.

\begin{tabular}{lcccc}
\hline$d_{p 2}$ & Group 2 & Group 3 & Group 4 & Group 5 \\
\hline Group 1 & 1 & 1 & 1 & 1 \\
Group 2 & & 0 & 0 & 0 \\
Group 3 & & & 0 & 0 \\
Group 4 & & & & 0 \\
\hline
\end{tabular}

objective, there was a difference between group 1 paired with each of the other groups, as shown in Table 4. We can further conclude that for the sensitive parameters, the variation in the responses between groups is different across parameters and groups. This does indicate that there may be some more complex interactions present.

5.4. Two-Way Interaction. In addition to main effects attributable to one parameter alone, many systems exhibit complex behavior that stems from nontrivial interactions between parameters. These can be visualized by interaction plots, which show the differences in effects between groups 

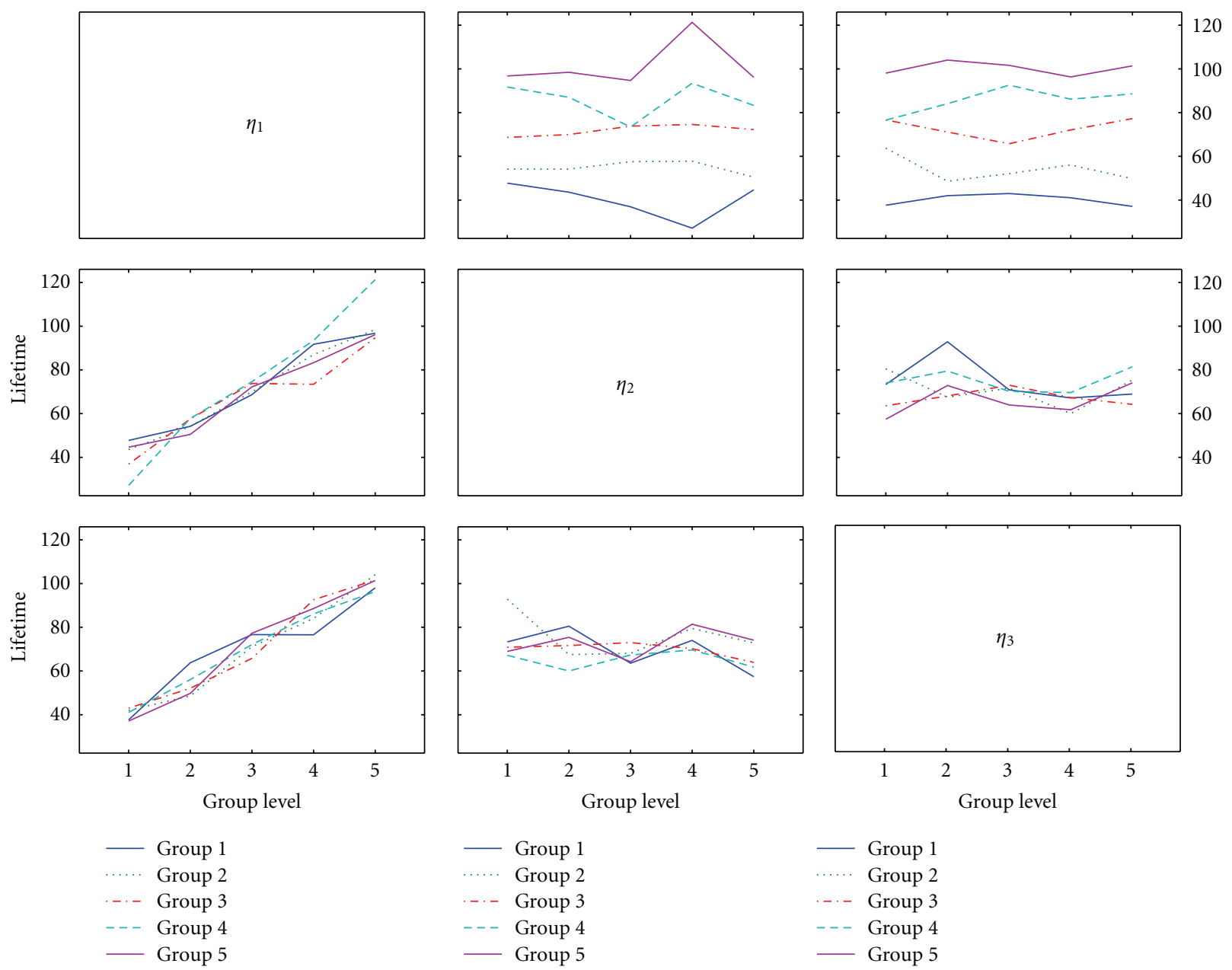

FIGURE 9: Interaction across all layers.

of one parameter and another as both are allowed to change. These plots take the form of one line for each group of a given parameter, plotted as the response function value when that parameter is fixed and another parameter varies over its groups. When these lines are nearly parallel, there are negligible interaction effects. That means changing one parameter has some effect regardless of the value of another. On the other hand, when the lines are at sharply different angles, especially when they cross, the conclusion may be drawn that the effect of changing one parameter depends strongly on the value of another. That interdependence is the manifestation of interaction effects. In this three-layer filter design analysis, there are six parameters, and thus there are 15 distinct two-way interaction possibilities for each objective function. Since each interaction can be plotted in two ways, there are 30 graphs in total. During the project, all of these were created, but we provide only some illustrative examples below.

Figure 8 shows the interaction of the porosity and pore diameter for layer one for the lifetime objective. For the top right portion of Figure $8(\mathrm{a})$, the horizontal axis is the quintile groups of average pore diameter in layer one. For its vertical axis, we show the average resulting filter lifetime. Each of the curves shown corresponds to a quintile level of porosity in layer one $\left(\eta_{1}\right)$. Thus, it can be seen that increasing porosity in layer one leads to increased filter life, as does increasing pore diameter in layer one. The plot in the bottom left corner of the same figure has for its horizontal axis the quintile groups of porosity in layer one. For its vertical axis, it also has the average resulting filter lifetime. But the curves correspond to quintile levels of average pore diameter in layer one. Thus, the two graphs show the same information in two different ways. In both cases, the lines are nearly parallel, which indicates little interaction between parameters. Thus, it does not matter what level of porosity is chosen, the effect of changing average pore diameter will be about the same, namely to increase filter lifetime.

The plots in Figure 8(b) show the same information, but for layer two of the filter. This time the lines are not parallel and do not tend to increase or decrease. Rather, there are sharp spikes and jagged lines. This indicates that there are substantial interaction effects manifested between the porosity and average pore diameter of layer two. For example, when the porosity is large, in Group 5, the lifetime could be very high if average pore diameter is in Group 4, or very low if average pore diameter is in Group 5. The effect of choosing 
a value for one variable strongly depends on the value of the other. The plots for layer three, although not shown here, have similarly strong interactions between porosity and pore diameter although the specific values of lifetime for a given pair of parameter levels are quite different from those seen for layer two.

The plot in the top right corner of Figure 9 has for its horizontal axis the quintile groups of porosity in layer three. For its vertical axis, it has the average resulting filter lifetime. Each of the curves shown corresponds to a quintile level of porosity in layer one. These curves do not have the frequent crossings seen above. However, the lines are not parallel, which indicates that changing one of these parameters could either increase or decrease the filter lifetime depending on the value of the other parameter. There are thus some interaction effects present between porosity in layer one and porosity in layer three. Each of the plots in that figure shows the interaction between porosity in some layer and porosity in another layer, and all exhibit noticeable effects. The plots with respect to the average pore diameters in each layer are similar to those above, but not shown here. Once again, substantial interaction effects are observed, with layers two and three especially having curves that cross repeatedly.

Plots analogous to the above, showing the responses in the escaped debris and secant objectives for the same interactions, were also generated. Overall, there are substantial interaction effects between nearly all parameters. This is to be expected, as the performance of one layer of a filter depends on what debris reaches it, which is determined by the performance of the upstream layers. Conversely, a downstream layer can compensate for upstream layers, thus sharing the load of debris and pressure drop, extending the life of the filter. In order to optimize the design of a polymer extrusion filter, parameters should be chosen that maximize lifetime and minimize both escaped debris and the secant objective. The main effects plots indicate how layer ought to be made, with large porosity and large average pore diameter. To discern how subsequent layers should be, it is necessary to consider interaction effects, looking for parameter combinations that achieve satisfactory values in each objective function.

\section{Conclusions}

We have considered both two-layer and three-layer polymer extrusion filter designs. Using a computational simulator, we assessed the design spaces to determine what parameters have significant effects. The two-layer results implied that only the first layer had significant effect on the performance metrics. By applying the Kruskal-Wallis ANOVA test, we rigorously showed that for both the lifetime and secant objectives, only first layer contributes for the three-layer model. For the escaped debris objective, the pore diameter in the second layer also contributes. The third layer had no main effects in any test we ran. There were interaction effects, but none on the scale of the main effects from the first layer.

These counterintuitive findings are open to several possible interpretations. On the one hand, if the simulator is accurate in its valuation of filter designs, then the first layer is paramount. A competitive filter design should be achievable with only one layer. Introducing a second layer may slightly decrease the total amount of debris that escapes but should not noticeably enhance the lifetime of the filter.

The simulator itself requires further study. While the initial results in [3] showed nice validation with empirical data, our sensitivity analysis gives results that seem to counter engineering understanding of the problem. This analysis guides us in reconciling these differences and enhancing the performance of our computational tool. This analysis has been important as it helped us better understand the filter design and the simulation tool, whose individual optimization studies and small comparisons with existing empirical data may not have uncovered.

\section{References}

[1] http://caeff.ces.clemson.edu/.

[2] C. L. Cox, E. B Duffy, and J. B. von Oehsen, "FISIM: the CAEFF integrated model for simulation of fiber and film processes," Plastics, Rubber \& Composites: Macromolecular Engineering, vol. 33, pp. 426-437, 2004.

[3] B. Seyfzadeh, D. A. Zumbrunnen, and R. A. Ross, "NonNewtonian flow and debris deposition in an extrusion filter medium," in Proceedings of the Plastics-The Lone Star, pp. 340-344, Society of Plastics Engineers, 2001.

[4] D. C. Hookway, "How to design your deep bed polymer filter," Filtration and Separation, vol. 33, no. 2, pp. 161-166, 1996.

[5] J. Markarian, “Choosing a melt filtration system," Plastics, Additives and Compounding, vol. 10, no. 3, pp. 32-35, 2008.

[6] K. R. Fowler, B. McClune, E. W. Jenkins, C. L. Cox, and B. Seyfzadeh, "Design analysis of polymer filtration using a multi-objective genetic algorithm," Separation Science and Technology, vol. 43, no. 4, pp. 710-726, 2008.

[7] K. R. Fowler, S. M. LaLonde, E. W. Jenkins, and C. L. Cox, "A simulation-based optimization approach to polymer extrusion filter design," in Proceedings of the American Filtration and Separations Society Annual Conference, American Filtration and Separations Society, 2008.

[8] K. R. Fowler, E. W. Jenkins, and S. M. LaLonde, "Understanding the effects of polymer extrusion filter layering configurations using derivative-free optimization," Optimization and Engineering, vol. 11, no. 2, pp. 339-354, 2010.

[9] K. R. Fowler, B. McClune, and E. W. Jenkins, "Polymer extrusion filter design with a hybrid pso-ga optimization technique," Filtration, vol. 11, no. 1, pp. 58-64, 2011.

[10] M. Van Parys, "PP fibre engineering," Chemical Fibers International, vol. 51, no. 5, pp. 317-319, 2001.

[11] D. D. Edie and C. H. Gooding, "Prediction of pressure drop for the flow of polymer melts through sintered metal filters," Industrial \& Engineering Chemistry Process Design and Development, vol. 24, no. 1, pp. 8-12, 1985.

[12] R. H. Christopher and S. Middleman, "Power-law flow through a packed tube," Industrial and Engineering Chemistry Fundamentals, vol. 4, no. 4, pp. 422-426, 1965.

[13] J. R. A. Pearson and P. M. J. Tardy., "Models for flow of non-Newtonian and complex fluids through porous media," Journal of Non-Newtonian Fluid Mechanics, vol. 102, pp. 447473, 2002.

[14] R. B. Bird, W. E. Stewart, and E. N. Lightfoot, Transport Phenomena, John Wiley \& Sons, New York, NY, USA, 1960.

[15] T. F. Russell and M. F. Wheeler, "Finite element and finite 
difference methods for continuous flows in porous media," in The Mathematics of Reservoir Simulation, R. E. Ewing, Ed., pp. 35-105, SIAM, Philadelphia, Pa, USA, 1983.

[16] B. S. Travis, N. Smith, J. O’Dwyer, and J. Camacho, "Discrete numerical modeling of flow through porous filter media," CAEFF REU poster.

[17] C. L. Cox, E. W. Jenkins, and P. J. Mucha, "Modeling of debris deposition in a polymer extrusion filter," in Proceedings of the PPS-21, Leipzig, Germany, June 2005. 

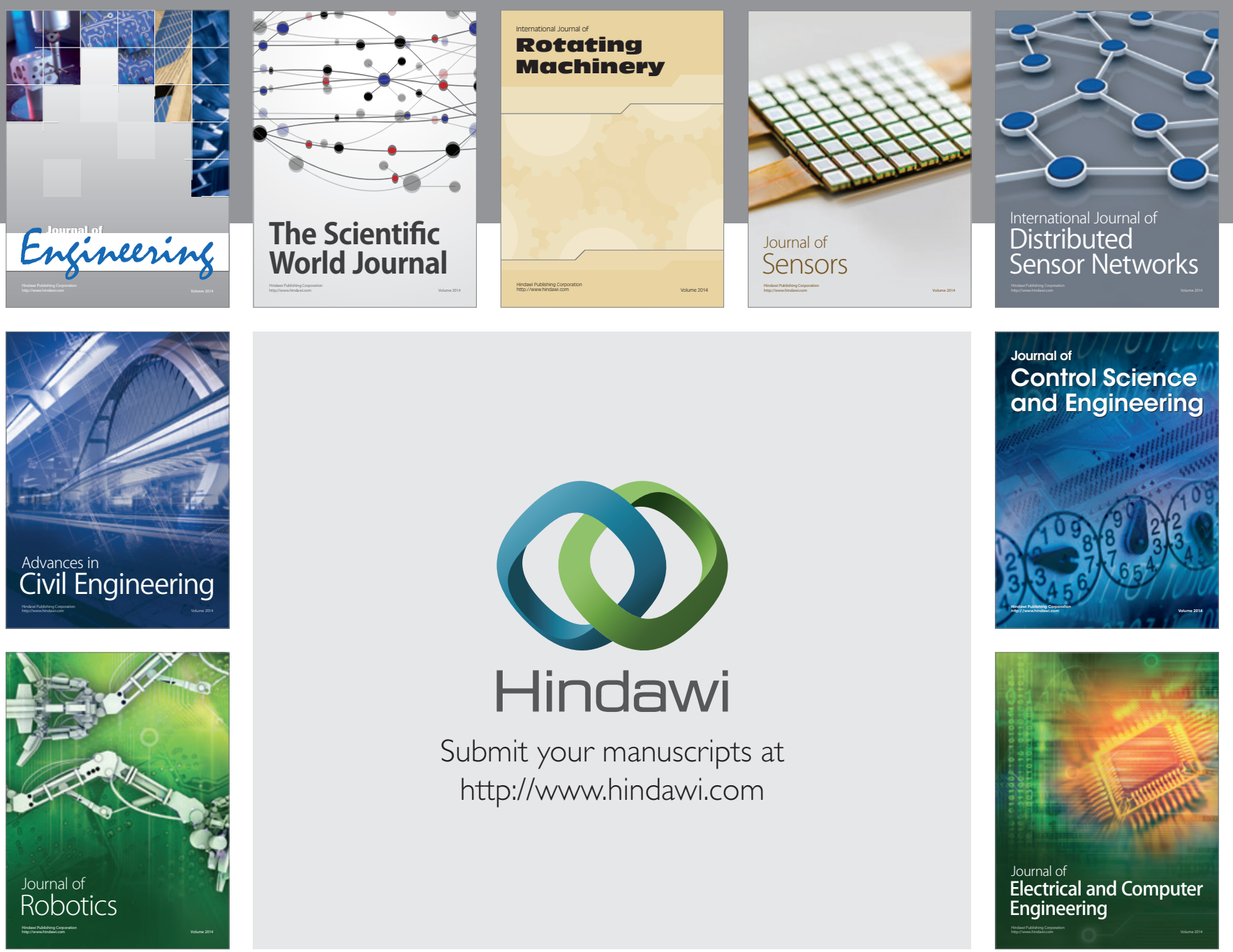

Submit your manuscripts at

http://www.hindawi.com
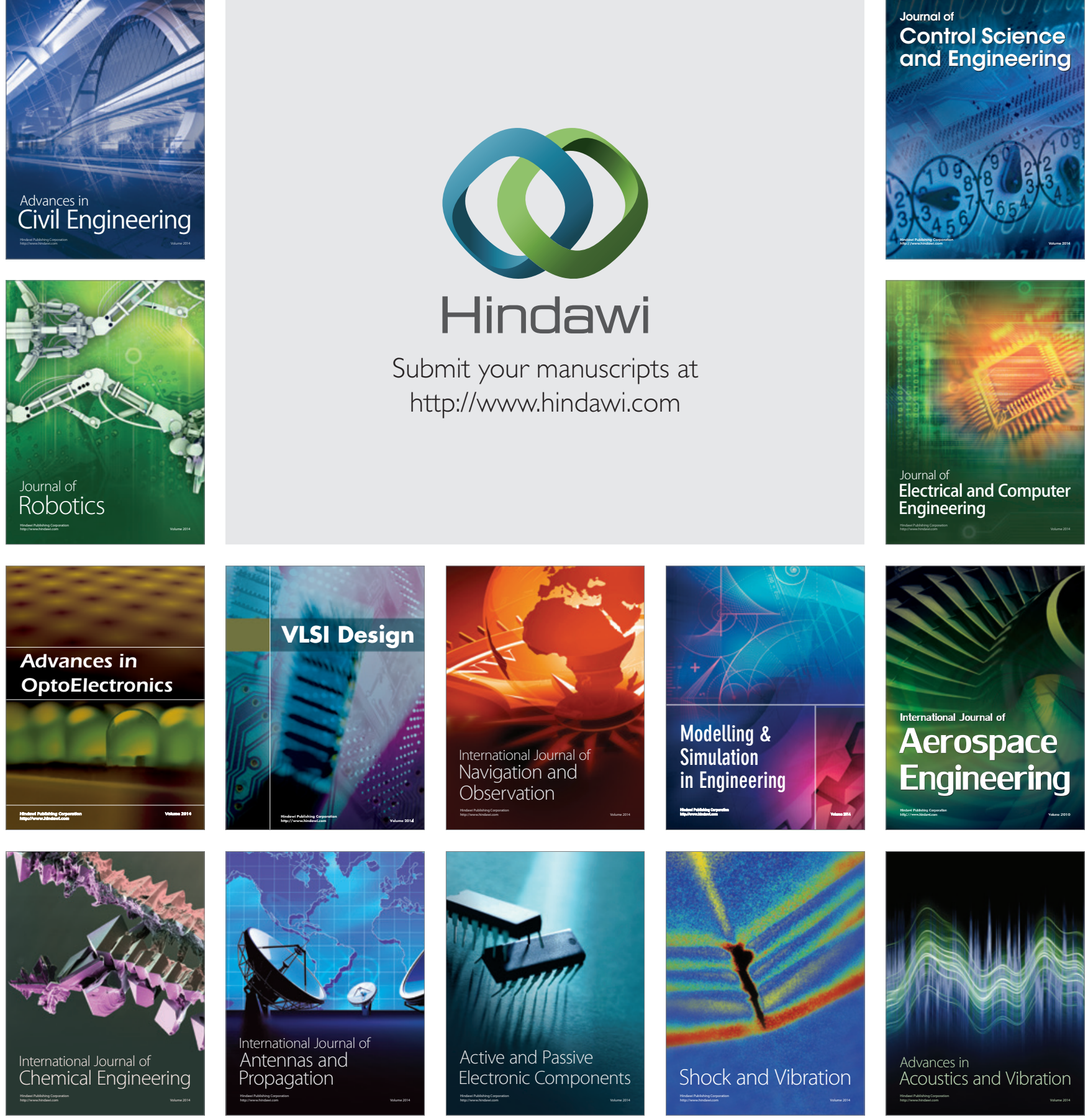